\title{
Reliability Evaluation of NPP's Power Supply System Based on Improved GO-FLOW Method
}

\author{
Jie Zhao, ${ }^{1}$ Tian Liu, ${ }^{1}$ Yu Zhao, ${ }^{1}$ Dichen Liu, ${ }^{1}$ Xiaodong Yang, ${ }^{2}$ \\ Yi Lin, ${ }^{2}$ Zhangsui Lin, ${ }^{2}$ and Yong Lei ${ }^{2}$ \\ ${ }^{1}$ School of Electrical Engineering, Wuhan University, Wuhan 430072, China \\ ${ }^{2}$ State Grid Fujian Electric Power Co. Ltd., Economic and Technology Institute, Fuzhou 350012, China \\ Correspondence should be addressed to Tian Liu; ltletitia@163.com
}

Received 21 June 2016; Revised 16 August 2016; Accepted 17 August 2016

Academic Editor: Eugenijus Ušpuras

Copyright (C) 2016 Jie Zhao et al. This is an open access article distributed under the Creative Commons Attribution License, which permits unrestricted use, distribution, and reproduction in any medium, provided the original work is properly cited.

NPP's power supply system is repairable and there is common cause failure between the components. The repair rate is introduced and total signaling is considered in the improved GO-FLOW method, aimed at reliability analysis for NPP's power supply system. Traditional GO-FLOW operators' algorithms are improved. Comprehensively considering the effect of total signaling flow in the power supply system, the equivalent reliability parameter model and common cause failure probability model of multimodal repairable components are constructed. The improved GO-FLOW model of NPP's power supply system is set up. Based on the proposed model, components' reliability parameters are computed. The failure probability time-varying trend in thirty years, respectively, of NPP's offsite power source and power supply system, is simulated and analyzed. Compared with calculation results of dynamic fault tree analysis method, the validity and the simplicity of the improved GO-FLOW method are verified. The effectiveness and applicability of the improved GO-FLOW model for NPP's power supply system are proved by simulation examples.

\section{Introduction}

Nuclear Power Plant's (NPP's) power supply system is the basis of nuclear safety as well as many control devices' reliable operation. It is generally composed of the main generator set, the offset main power supply system, auxiliary power supply system, reserved diesel generator set, and uninterruptible power system (UPS). Reliability evaluation aimed at NPP's power supply system helps find out vulnerable spots, and targeted measures are formulated to improve safety and reliability.

At present, the reliability analysis methods mainly include the fault tree method [1], Markov Method [2], Monte Carlo Method [3], and the GO-FLOW method [4]. When applied in the reliability evaluation of NPP with substantial scale and complex structure, these methods all have certain limitations. NPP's power supply system, which contains a large quantity of devices, has complex structure. It is also a dynamic system with complex phased operation sequence and time-varying states [5]. Currently, statistics and engineering methods are adopted to analyze failure probability when the reliability of NPP's power supply system is studied [6]. This kind of method needs mass of statistical data and has many limitations. The dynamic fault tree (DFT) method is available in the reliability evaluation despite relatively complicated calculations [7]. However, GO-FLOW method can directly simulate system [8]. In the GO-FLOW method, components' operator algorithm models are established based on structure characteristics and operation mechanism of systems. GO-FLOW method can effectively solve the probability analysis, when used in dynamic system with time sequence and phased mission [9]. It has been applied in PWR's containment spray system [10], risk monitoring and control system [11], automatic decompression system [12], residual heat removal system [13], and some other systems. GO-FLOW method has effectively completed the quantitative evaluation of dynamic reliability [14]. When GO-FLOW method is used in NPP's power supply system, GO-FLOW models are established, and equivalent parameters are calculated, reliability calculation can be achieved without reliance on mass of statistical data [15]. 


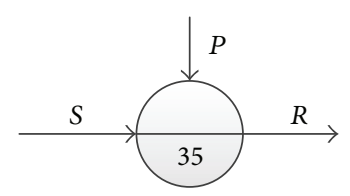

(a) Type 35 operator

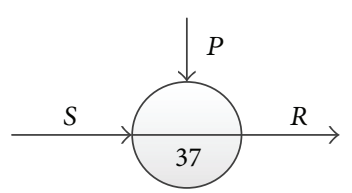

(b) Type 37 operator

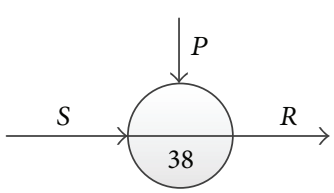

(c) Type 38 operator

FIGURE 1: GO-FLOW operators commonly used.

However, NPP's power supply system is repairable and there is common cause failure between the components. There is also multiple total signaling in the power supply system. Calculation error is inevitable when these factors are ignored. Besides, most of the components, which have at least two kinds of failure modes or maintenance modes, are multimodal. The redundancy design of important systems makes common cause failure between components easily happen. The existing literature [16] simply discusses equivalent reliability parameters of components, neither considering devices' maintenance condition nor dealing with total signaling in the algorithm model. Generally, when GO-FLOW method is applied in reliability evaluation of NPP's power supply system, pertinent improvement should be promoted in order to achieve better effect and more accurate results.

Improved GO-FLOW method is proposed for reliability analysis of NPP's power supply system, with common cause failure factors considered and total signaling [17] and components' repair rates [18] taken into account. Components' operator algorithm models are improved. Based on the algorithm, improved GO-FLOW model of NPP's power supply system is established. And the failure probability of NPP's offsite power as well as its power supply system is calculated. Comparison analysis of the DFT method and the improved GO-FLOW method verified the effectiveness of improved GO-FLOW method. Besides, the improved GO-FLOW method is contrasted with GO-FLOW method considering neither the repair rate nor the total signaling, or ignoring one of them. The accuracy and applicability of the improved GO-FLOW method can be shown through multiple contrast analysis.

\section{Repairable Components' Algorithm Model Based on Improved GO-FLOW Method}

2.1. Repairable Components' Fundamental Assembled Algorithm Model. When the GO-FLOW method is adopted to analyze system's reliability, to begin with, system's schematic diagram should be converted into GO-FLOW diagram by GO-FLOW operators. Operators' algorithm rule of existing GO-FLOW method does not consider maintenance case. Therefore, when GO-FLOW method is applied in repairable system, it is demanded that GO-FLOW operators be improved firstly.

GO-FLOW method defines fourteen types of normative operators [19], which are classified into three types, namely, function operators, logic operators, and signal generator operators. Function operators are used to simulate physical

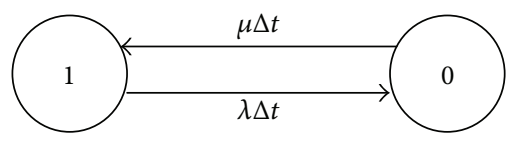

FIGURE 2: States transition diagram for components with two states.

components in systems, representing the working or failure states of components. Logic operators simulate the logical relationship between components. Signal generator operators simulate the external input signal in systems.

For example, when type 35 operator (shown in Figure 1(a)) is used to describe repairable components with two states, repair rate $\mu$ is demanded to be introduced to improve this operator. States transition diagram for components with two states is shown in Figure 2. In the figure, "1" represents success state and " 0 " represents failure state.

Based on Markov states transition theory, component's success probability from time $t$ to time $(t+\Delta t)$ can be deduced:

$$
\begin{aligned}
P_{1}(t+\Delta t)= & \frac{\mu}{\lambda+\mu} \\
& -\frac{\mu-(\lambda+\mu) P_{1}(t)}{\lambda+\mu} \exp [-(\lambda+\mu) \Delta t],
\end{aligned}
$$

where $P_{1}(t)$ is component's success probability in time $t, P_{1}(t+$ $\Delta t)$ is component's success probability in time $(t+\Delta t), \lambda$ is component's failure rate, and $\mu$ is component's repair rate.

If type 35 operator is used to represent normally closed electric valve, which is a typical repairable component, assembled model is shown in Figure 3, and algorithm is shown as

$$
\begin{aligned}
& R(t)=S(t)\left\{\frac{\mu}{\lambda+\mu}-\frac{\mu-(\lambda+\mu) P_{1}(t)}{\lambda+\mu}\right. \\
& \cdot \exp [-(\lambda+\mu) \\
& \left.\left.\cdot \sum_{i} \sum_{t_{k} \leq t} P_{i}\left(t_{k}\right) \cdot \min \left(1.0, \frac{S\left(t_{k}\right)}{S(t)}\right)\right]\right\},
\end{aligned}
$$

where $R(t)$ is output signal's intensity of operator $n_{2}, P_{i}\left(t_{k}\right)$ is the secondary input signals at intervals, $i$ is the number of the secondary input signals $P_{i}\left(t_{k}\right), t$ is the time point, $t_{k}$ is the $k$ th point in time, $S(t)$ is input signal's intensity of operator $n_{1}$ at time point $t, S\left(t_{k}\right)$ is input signal's intensity of operator $n_{1}$ at 


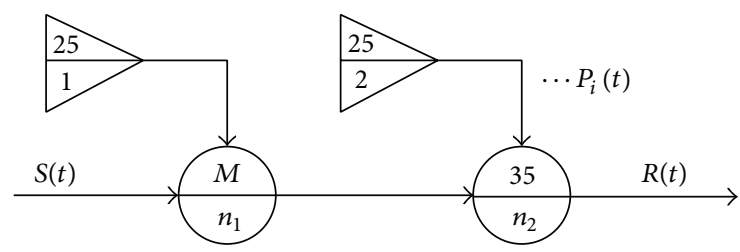

FIgUre 3: Assembled GO-FLOW model for repairable component.

time point $t_{k}$, and $P_{1}(t)$ is success probability of operator $n_{1}$ at time point $t$.

Operator $n_{1}$ should be selected depending on the type of components. For instance, type 26 operator is selected to represent operator $n_{1}$ for normally closed electric valve. Operator $n_{2}$ is type 35 operator, which is used to represent states changes caused by failure rate and repair rate during components' operation process. And the output signal intensity is components' success probability with maintenance condition considered. The assembled algorithm rule of operator $n_{1}$ and operator $n_{2}$ is comprehensively represented in (2). When $\mu=0$, it is a particular case with repair rate not considered as shown in (2). In consequence, the assembled algorithm rule represented in (2) is universal, applicable to both repairable systems and irreparable systems.

When type 35 operator in Figure 3 is replaced by type 37 operator (shown in Figure 1(b)), assembled model of type 37 operator with repair rate considered can be deduced. Assembled operation algorithm is shown in

$$
\begin{aligned}
& R(t)=S(t)\left\{\frac{\mu}{\lambda+\mu}\right. \\
& \left.-\frac{\mu-(\lambda+\mu) P_{1}(t)}{\lambda+\mu} \exp \left[-(\lambda+\mu) \sum_{i} \sum_{t_{k} \leq t} P_{i}\left(t_{k}\right)\right]\right\} .
\end{aligned}
$$

Similarly, assembled model of type 38 operator (shown in Figure 1(c)) with repair rate considered can be deduced. Assembled operation algorithm is shown in

$$
\begin{gathered}
R(t)=S(t)\left\{1-\frac{\mu}{\lambda+\mu}-\frac{\mu-(\lambda+\mu) P_{1}(t)}{\lambda+\mu}\right. \\
\left.\cdot \exp \left[-(\lambda+\mu) \sum_{i} \sum_{t_{k} \leq t} P_{i}\left(t_{k}\right)\right]\right\} .
\end{gathered}
$$

2.2. The Equivalent Model for Reliability Parameters of Multimodal Repairable Components. For repairable components with two failure modes, there may be three types of states, namely, the normal working state, the maintenance state during the first failure mode, and the maintenance state during the second failure mode, which can be, respectively, represented by " 0 ," "1," and " 2 ," as shown in Figure 4.

In Figure $4, \lambda_{1}$ and $\mu_{1}$, respectively, represent the failure rate and repair rate of the first failure mode. $\lambda_{2}$ and $\mu_{2}$, respectively, represent the failure rate and repair rate of the second failure mode. The component' states transition equation is

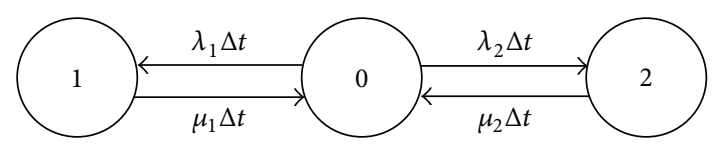

FIGURE 4: States transition diagram for repairable component with two failure modes.

shown in (5). If the probability for components' three types of states is known at the initial time $t$, the probabilities $P_{0}(t+\Delta t)$, $P_{1}(t+\Delta t)$, and $P_{2}(t+\Delta t)$ for components' all types of states at any time $(t+\Delta t)$ can be achieved:

$$
\begin{aligned}
& {\left[\begin{array}{c}
P_{0}(t+\Delta t) \\
P_{1}(t+\Delta t) \\
P_{2}(t+\Delta t)
\end{array}\right]} \\
& =\left(\begin{array}{ccc}
1-\lambda_{1} \Delta t-\lambda_{2} \Delta t & \mu_{1} \Delta t & \mu_{2} \Delta t \\
\lambda_{1} \Delta t & 1-\mu_{1} \Delta t & 0 \\
\lambda_{2} \Delta t & 0 & 1-\mu_{2} \Delta t
\end{array}\right)\left[\begin{array}{l}
P_{0}(t) \\
P_{1}(t) \\
P_{2}(t)
\end{array}\right],
\end{aligned}
$$

where $P_{0}(t), P_{1}(t)$, and $P_{2}(t)$, respectively, represent the probability of components' states " 0 ," " 1 ," and " 2 " at time $t$. Meanwhile, the relationship between $P_{0}(t), P_{1}(t)$, and $P_{2}(t)$ is as follows:

$$
P_{0}(t)+P_{1}(t)+P_{2}(t)=1 \text {. }
$$

For a repairable component with two types of failure modes, it can be equivalent to a repairable component with just one type of failure mode by type 21 two-state operator. The equivalent reliability parameters of the operator are represented in (7). Maintenance state can be regarded as a particular type of failure state:

$$
\begin{aligned}
& P_{c}(t)=P_{0}(t), \\
& \lambda_{c}(t)=\lambda_{1}+\lambda_{2}, \\
& \mu_{c}(t)=\frac{\mu_{1} P_{1}(t)+\mu_{2} P_{2}(t)}{1-P_{0}(t)},
\end{aligned}
$$

where $P_{c}(t)$ is the equivalent failure probability of repairable components and $\lambda_{c}$ and $\mu_{c}$, respectively, represent the equivalent failure rate and repair rate of repairable components.

In a system with $n$ repairable components, when the influences caused by common cause failure between repairable components are considered, common cause failure probability $C_{n}(t)$ at time $t$ is approximately represented in

$$
\begin{aligned}
C_{n}(t)= & \frac{c}{c+\sum_{i=1}^{n} \mu_{i}} \\
& +\left[\gamma_{c}-\frac{c}{c+\sum_{i=1}^{n} \mu_{i}}\right] \exp \left[-\left(c+\sum_{i=1}^{n} \mu_{i}\right) t\right],
\end{aligned}
$$

where $c$ is the probability of common cause failure and $\gamma_{c}$ is the probability of system's common cause failure at initial moment. 


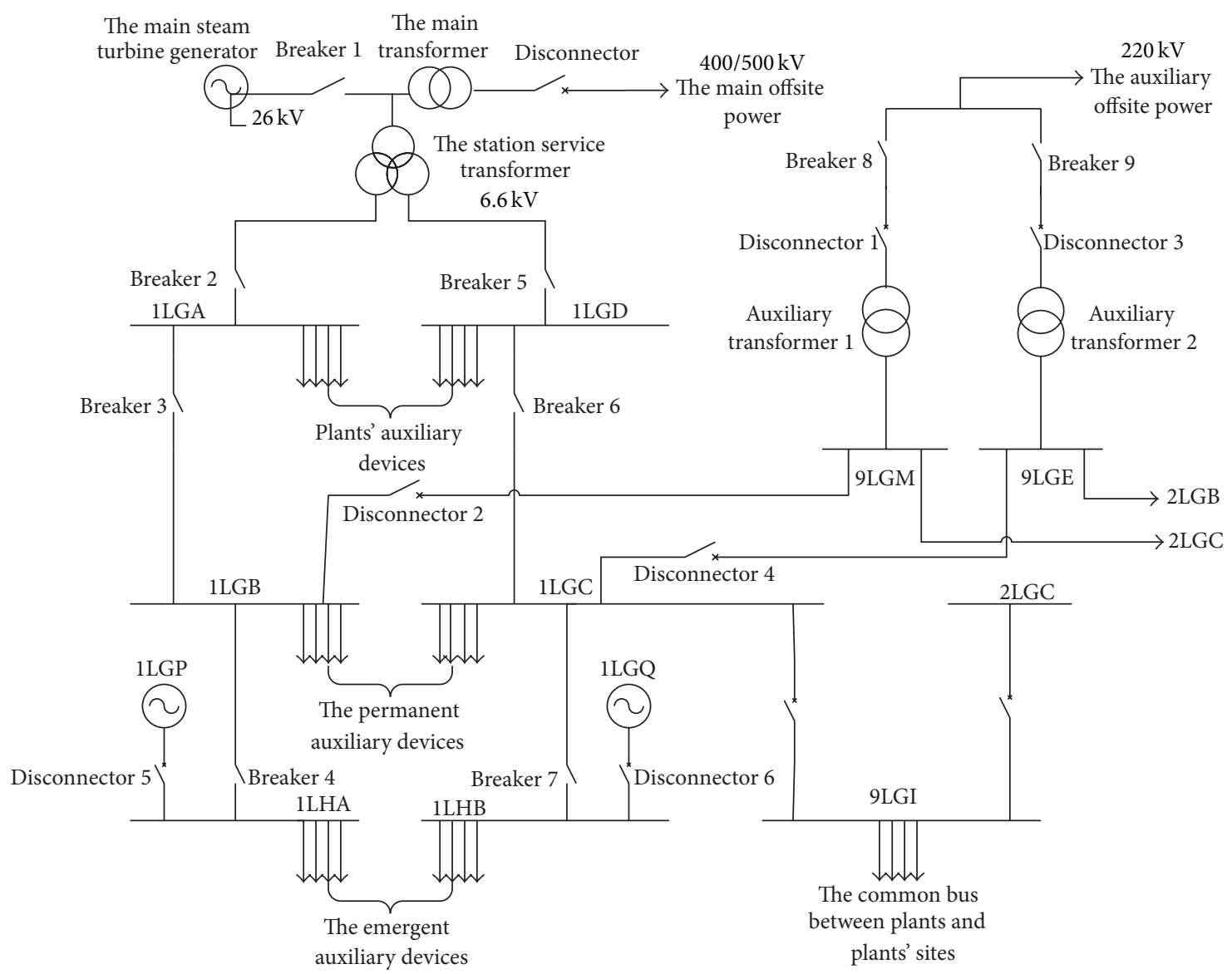

FIGURE 5: Diagram of NPP's power supply system.

2.3. Improved Quantitative Calculation Method considering Total Signaling. For the point of time with two total signals, denoted as $a$ and $b$, the existing probability of the output signal is revised with total signaling considered. General form of the expression containing total signaling items is as follows:

$$
R_{f}=C_{0}+C_{1} R_{a}+C_{2} R_{b}+C_{3} R_{a} R_{b},
$$

where $R_{a}$ and $R_{b}$ are, respectively, the existing probability of signal $a$ and signal $b$, which can be calculated through the GO-FLOW method. $R_{f}$ is the existing probability of system's terminal signal $f . C_{0}, C_{1}, C_{2}$, and $C_{3}$, which are constant, are correction coefficients irrelevant to the total signaling.

Total signals $a$ and $b$ in the system are, respectively, set up as four assembled states, namely, failure-failure $\left(R_{a}=0\right.$, $\left.R_{b}=0\right)$, failure-success $\left(R_{a}=0, R_{b}=1\right)$, success-failure $\left(R_{a}=1, R_{b}=0\right)$, and success-success $\left(R_{a}=1, R_{b}=1\right)$. The values of $R_{f}$ in these four states are, respectively, $R_{f 00}, R_{f 01}$, $R_{f 10}, R_{f 11}$, which are irrelevant to the total signaling.

Substituting $R_{f 00}, R_{f 01}, R_{f 10}, R_{f 11}$ in (9), (9) is transformed to the next equation:

$$
\begin{aligned}
R_{f}= & \left(1-R_{a}\right)\left(1-R_{b}\right) R_{f 00}+R_{a}\left(1-R_{b}\right) R_{f 10} \\
& +\left(1-R_{a}\right) R_{b} R_{f 01}+R_{a} R_{b} R_{f 11},
\end{aligned}
$$

where $R_{f 00}=C_{0}, R_{f 01}=C_{0}+C_{2}, R_{f 10}=C_{0}+C_{1}$, and $R_{f 11}=C_{0}+C_{1}+C_{2}+C_{3}$.
If there are $L$ total signals in the system, respectively, described as $S_{l}(l=1,2, \ldots, L)$, the existing probability of $S_{l}$ is $R_{S_{l}}(l=1,2, \ldots, L)$. Similarly, the existing probability of the terminal signal $R_{f}$ is as follows:

$$
\begin{aligned}
& R_{f} \\
& =\sum_{K_{1}=0}^{l} \sum_{K_{2}}^{l} \cdots \sum_{K_{L}=0}^{l} R_{f K_{1} K_{2} \cdots K_{L}} \prod_{l=1}^{L}\left[\left(1-R_{S_{l}}\right)\left(1-K_{l}\right)+R_{S_{l}} K_{l}\right] .
\end{aligned}
$$

\section{Model for NPP's Power Supply System Based on Improved GO-FLOW Method}

3.1. The GO-FLOW Model for NPP's Power Supply System. The Daya bay NPP's power supply system (shown in Figure 5) is taken as an example. During the normal operation of NPP, power is supplied by $26 \mathrm{kV}$ bus through a HV station service step-down transformer. For the $26 \mathrm{kV}$ bus, power is supplied by main generator during the operation of NPP and by UHV main offsite grid (400 kV Hong Kong grid/500 kV Guangdong grid) through the main transformer during NPP's shutdown. When $26 \mathrm{kV}$ bus loses power, $220 \mathrm{kV}$ offsite auxiliary power supply is switched to supply power to the permanent bus, the emergency bus, and the common bus, with 1.5 3 s' time delay. When the generator set, the main 


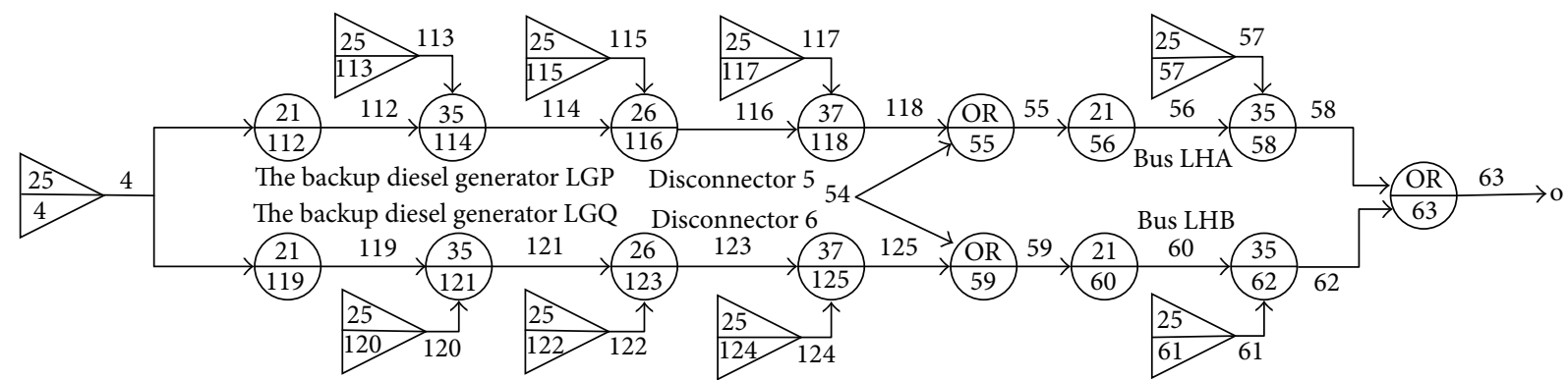

(a) The GO-FLOW diagram of NPP's reserved system
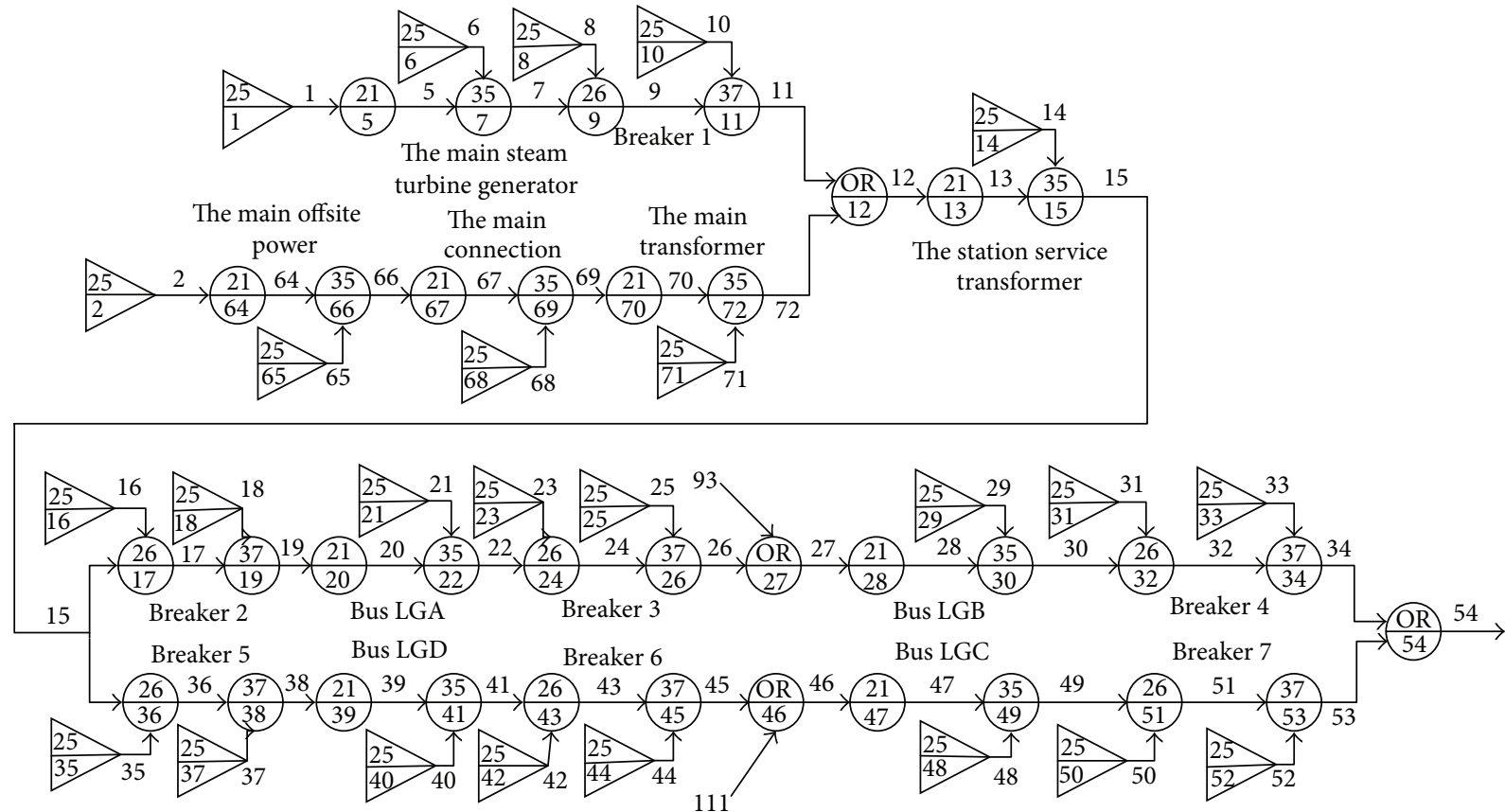

(b) The GO-FLOW diagram of NPP's main power supply system



(c) The GO-FLOW diagram of NPP's auxiliary power supply system

FIGURE 6: The GO-FLOW diagram of NPP's power supply system.

offsite power supply, and the auxiliary offsite power supply are all unavailable, backup diesel generator set supplies power through the emergency bus, and the NPP gets into a state of cold shutdown. The backup diesel generator sets are represented as LGP and LGQ.
The three modules' GO-FLOW diagrams of NPP's power supply system are set up dividedly, as shown in Figure 6:

(1) Components are simulated by the assembled operator algorithm models for repairable components. 
TABLE 1: The improved GO-FLOW method used in NPP's power supply system.

\begin{tabular}{|c|c|c|c|}
\hline Item & $\begin{array}{l}\text { The existing } \\
\text { GO-FLOW } \\
\text { method }\end{array}$ & $\begin{array}{l}\text { The improved } \\
\text { GO-FLOW method }\end{array}$ & The quantitative calculation method after being improved \\
\hline $\begin{array}{l}\text { Considering components' } \\
\text { repair rate or not }\end{array}$ & No & $\begin{array}{l}\text { GO-FLOW operator } \\
\text { algorithm is improved } \\
\text { by introducing repair } \\
\text { rate }\end{array}$ & $\begin{array}{l}\text { For example, the success probability of components with two states } \\
\text { at time }(t+\Delta t) \text { is as } \\
P_{1}(t+\Delta t)=\frac{\mu}{\lambda+\mu}-\frac{\mu-(\lambda+\mu) P_{1}(t)}{\lambda+\mu} \exp [-(\lambda+\mu) \Delta t]\end{array}$ \\
\hline $\begin{array}{l}\text { Considering total signaling } \\
\text { or not }\end{array}$ & No & $\begin{array}{l}\text { Synthesize the effect } \\
\text { of total signaling }\end{array}$ & $\begin{array}{l}\text { The existing probability of the terminal signal in a system with } L \\
\text { total signals is as } \\
R_{f}=\sum_{K_{1}=0}^{l} \sum_{K_{2}}^{l} \cdots \sum_{K_{L}=0}^{l} R_{f K_{1} K_{2} \cdots K_{L}} \prod_{l=1}^{L}\left[\left(1-R_{S_{l}}\right)\left(1-K_{l}\right)+R_{S_{l}} K_{l}\right]\end{array}$ \\
\hline $\begin{array}{l}\text { Considering common } \\
\text { cause failure or not }\end{array}$ & Yes & Yes & $\begin{array}{l}\text { At time } t \text {, the common cause failure probability of a system with } n \\
\text { repairable components is as } \\
C_{n}(t)=\frac{c}{c+\sum_{i=1}^{n} \mu_{i}}+\left[\gamma_{c}-\frac{c}{c+\sum_{i=1}^{n} \mu_{i}}\right] \exp \left[-\left(c+\sum_{i=1}^{n} \mu_{i}\right) t\right]\end{array}$ \\
\hline $\begin{array}{l}\text { Applicable to complex } \\
\text { repairable system with } \\
\text { multistates or not }\end{array}$ & $\begin{array}{l}\text { Calculation } \\
\text { error }\end{array}$ & $\begin{array}{l}\text { Much more } \\
\text { reasonable results }\end{array}$ & $\begin{array}{l}\text { For example, states transition equation of repairable components } \\
\text { with three states is as } \\
P_{0}(t+\Delta t)=\left(1-\lambda_{1} \Delta t-\lambda_{2} \Delta t\right) P_{0}(t)+\mu_{1} \Delta t P_{1}(t)+\mu_{2} \Delta t P_{2}(t) \\
P_{1}(t+\Delta t)=\lambda_{1} \Delta t P_{0}(t)+\left(1-\mu_{1} \Delta t\right) P_{1}(t) \\
P_{2}(t+\Delta t)=\lambda_{2} \Delta t P_{0}(t)+\left(1-\mu_{2} \Delta t\right) P_{2}(t) \\
P_{0}(t)+P_{1}(t)+P_{2}(t)=1\end{array}$ \\
\hline
\end{tabular}

(2) Failure rate and repair rate of repairable conductive components can be set up according to actual parameters.

(3) Assume that components in the system are all repairable components submitted to exponential distribution, the failure rate and repair rate of which are constant.

(4) Repairable components are represented with type 21 operator and 35 operator cascaded and connected by signal flow.

(5) The outputs of buses 9LGM and 9LGE in the auxiliary circuit are connected with the inputs of buses LGB and LGC in the main circuit through type 22,27 , and 46 operator.

(6) The outputs of the station service transformer are, respectively, connected with buses LGA and LGB and buses LGD and LGC, to form two main power supply circuits. When buses LGB and LGC do not supply power, NPP loses the offsite power, namely, LOOP accident. When the reserved system's output signal 63 faults, buses LHA and LHB2 do not supply power; NPP loses all power, namely, SBO accident.

3.2. The Analysis Process of Improved GO-FLOW Method. The improvements of GO-FLOW method when applied in NPP's power supply system are shown in Table 1.

The process when improved GO-FLOW method is applied in the reliability analysis of NPP's power supply system is shown in Figure 7, concretely as follows:

(1) Select the GO-FLOW operators according to the structure of NPP's power supply system and characters of its components. Improve the repairable components' algorithm by introducing components' repair rates. Establish the reliability parameters' equivalent model for multimodal repairable components. Get the analog parameter model and state probability of components with various failure modes.

(2) Select reliability indexes of NPP's power supply system, namely, failure frequency, steady-state value of failure probability, failure duration, mean repairing time and maintenance frequency, and maintenance time. Synthesize the reliability indexes, the reliability parameters, equivalent failure rate, equivalent repair rate, mean success probability, and mean failure probability that can be, respectively, achieved.

(3) Confirm the functional and the logical relationship between every unit. Comprehensively consider the influence of total signaling in the NPP's power supply system. Establish the GO-FLOW diagram for the NPP's power supply system. And connect GO-FLOW operators by signal flow. Number the operators and the signal flows.

(4) Analyze NPP's LOOP/SBO accident, and ascertain system's inputs and outputs.

(5) Define a finite number of discrete time points to represent the processing sequence, and input the state probability data of every unit.

(6) Compute until outputting signals. Calculate system's reliability and analyze the results. Evaluate the system according to its functions and demands. 


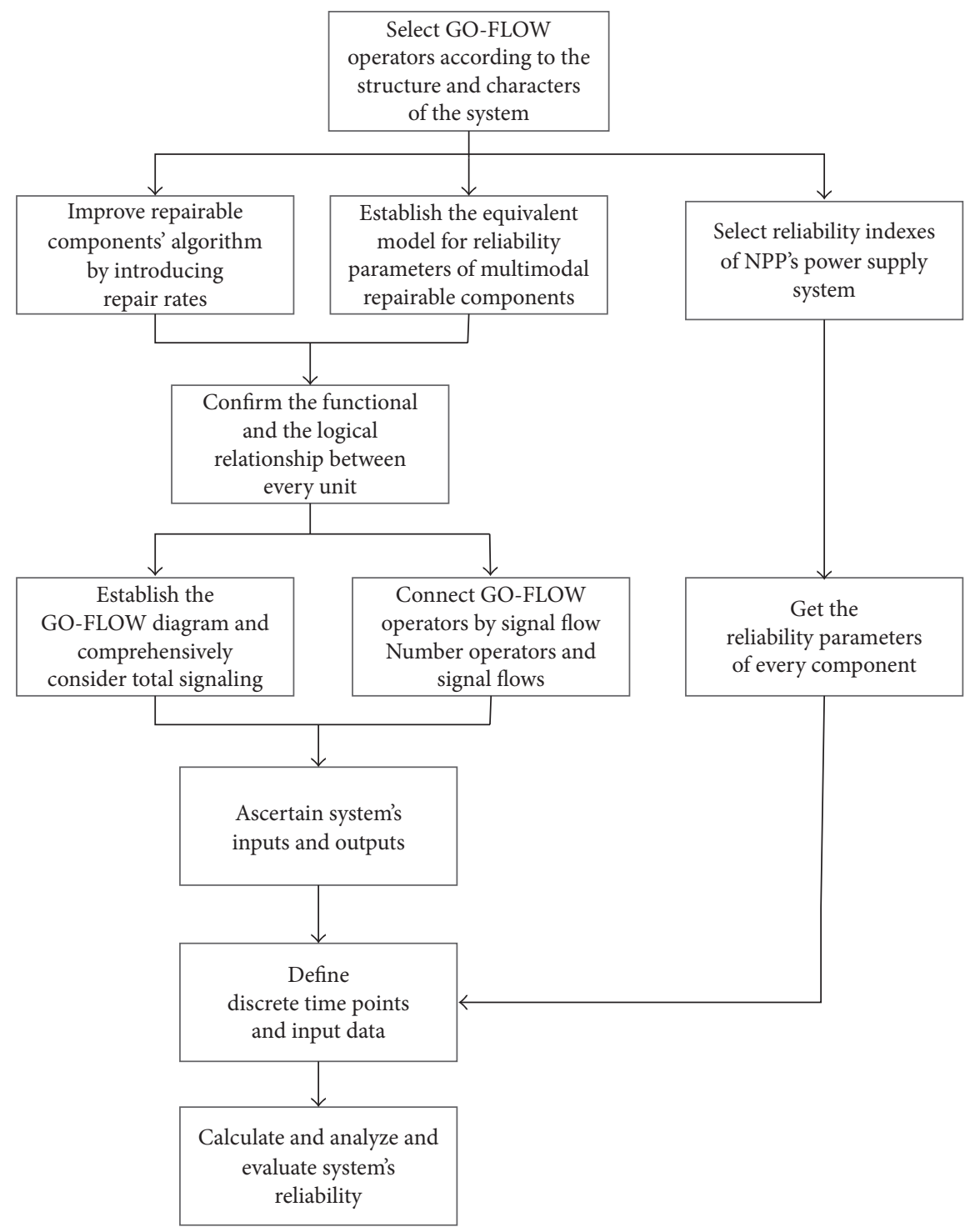

FIGURE 7: The GO-FLOW analysis process diagram of NPP's power supply system.

\section{Simulation and Calculation}

4.1. Calculation Case. Simulation is completed based on the improved GO-FLOW model of NPP's power supply system. Meanwhile compare with the DFT method.

Confirm system's failure modes which definitely develop into a fault discussed. Based on analog components' multimodal failure behavior and dynamic gate's success criteria, dynamic fault tree for NPP's power supply system is built. Reliability analysis and outage probability calculation for NPP's power supply system, which contains multimodal failure behavior, are simulated by the DFT method.

Simulate and analyze the failure probability as well as its time-varying trend of NPP's offsite power and power supply system. Reliability parameters are selected, as shown in Tables 2 and $3[20,21]$. Regard the disconnector as a component whose success probability is always 1 . Define the computation time as 30, on account that the operating life of a nuclear reactor is generally 30 years. The step length is 1 (i.e., 1 year).

4.2. Reliability Analysis of NPP's Offsite Power System. The failure probability of NPP's offsite power system changes with time, as shown in Figure 8.

Compare and analyze the calculation results of the four concerned methods, namely, the DFT method, the GOFLOW method considering neither repair rate nor total signaling, the GO-FLOW method considering repair rate but not total signaling, and the GO-FLOW method considering repair rate and total signaling. As is shown in Figure 8, we have the following:

(1) When the repair rate and the total signaling are not taken into consideration, the failure probability of NPP's offsite power system increases year by year with 
TABLE 2: The failure frequency and mean repairing time of repairable components.

\begin{tabular}{|c|c|c|c|}
\hline Names of the units & Number of failure modes & $\begin{array}{l}\text { Failure frequency } \\
\text { (times per year) }\end{array}$ & $\begin{array}{c}\text { Mean repairing time } \\
\text { (hours) }\end{array}$ \\
\hline The main steam turbine generator & 1 & $1.500 e+00$ & $7.300 e+01$ \\
\hline The main offsite power & 1 & $2.300 e-03$ & $1.000 e+02$ \\
\hline The auxiliary offsite power & 1 & $8.468 e+00$ & $3.351 e+00$ \\
\hline Reserved diesel generator & 1 & $1.743 e+02$ & $5.000 e+00$ \\
\hline \multirow{2}{*}{ The main transformer } & \multirow[t]{2}{*}{2} & $2.015 e-03$ & $1.000 e+01$ \\
\hline & & $8.760 e-03$ & $7.500 e+02$ \\
\hline \multirow{2}{*}{ The station service transformer } & \multirow{2}{*}{2} & $5.694 e-03$ & $1.000 e+01$ \\
\hline & & $8.760 e-03$ & $4.000 e+02$ \\
\hline \multirow{2}{*}{ The auxiliary transformer } & \multirow{2}{*}{2} & $1.139 e-02$ & $1.000 e+01$ \\
\hline & & $1.139 e-02$ & $4.000 e+02$ \\
\hline The main connection & 1 & $2.059 e-02$ & $1.060 e+02$ \\
\hline \multirow{2}{*}{ Bus } & \multirow{2}{*}{2} & $3.679 e-03$ & $5.000 e+00$ \\
\hline & & $4.643 e-04$ & $7.200 e+01$ \\
\hline Breaker of generator circuit & 1 & $4.890 e-02$ & $1.116 e+02$ \\
\hline Breaker & 1 & $3.030 e-02$ & $5.750 e+01$ \\
\hline Disconnector & 1 & 0 & 0 \\
\hline
\end{tabular}

TABLE 3: The maintenance frequency and maintenance time of repairable components.

\begin{tabular}{lccc}
\hline Names of the units & States of the units & $\begin{array}{c}\text { Maintenance frequency } \\
\text { (times per year) }\end{array}$ & $\begin{array}{c}\text { Maintenance time } \\
\text { (hours) }\end{array}$ \\
\hline The main steam turbine generator & Maintenance & $1.000 e+00$ & $2.800 e+02$ \\
The main transformer & Maintenance & $2.000 e-01$ & $1.600 e+02$ \\
The main connection & Maintenance & $5.000 e-01$ & $2.400 e+01$ \\
Breaker & Maintenance & $7.872 e-01$ & $8.100 e+01$ \\
\hline
\end{tabular}

the operation time. When the repair rate and the total signaling are taken into consideration, within the five years after NPP is put into operation, the failure probability of NPP's offsite power system increases obviously due to the increase of components' failure rate, while, five years later, the failure probability of NPP's offsite power system gradually flattens out due to components' high repair rate and regular maintenance.

(2) The failure probability of NPP's offsite power system calculated by GO-FLOW method regardless of total signaling is about $5 \%$ lower than that calculated by GO-FLOW method with total signaling considered.

(3) The failure probability of NPP's offsite power system calculated by improved GO-FLOW method (considering both repair rate and total signaling) has the same time-varying trend with that calculated by DFT method. It shows that the improved GO-FLOW method can effectively calculate failure probability of offsite power system. The applicability of improved GO-FLOW method in complex multistate repairable system is verified.

(4) The failure probability of NPP's offsite power system calculated by improved GO-FLOW method is about
$10 \%$ higher than that calculated by DFT method. Since that, the fault tree method is oriented to system's failure. Its quantitative analysis is simplified by the minimal cut sets' independent approximation and high-order cut sets' truncation. Error increases with the number of components' states. Nevertheless, the improved GO-FLOW method is oriented to system's success. When it is applied in complex multistate system, the failure probability is much more accurate because the simplified approximation which is previously mentioned is avoided.

4.3. Reliability Analysis of NPP's Power Supply System. The failure probability of NPP's power supply system changes with time, as shown in Figure 9.

Figure 9 shows the following:

(1) The failure probability of NPP's power supply system, whose magnitude is -7 , is two orders of magnitude higher than that of NPP's offsite power system, because components' repair rate is much higher than the failure rate in NPP's power supply system. And the backup diesel generator sets can be put into operation to supply power to the emergency bus when needed. 


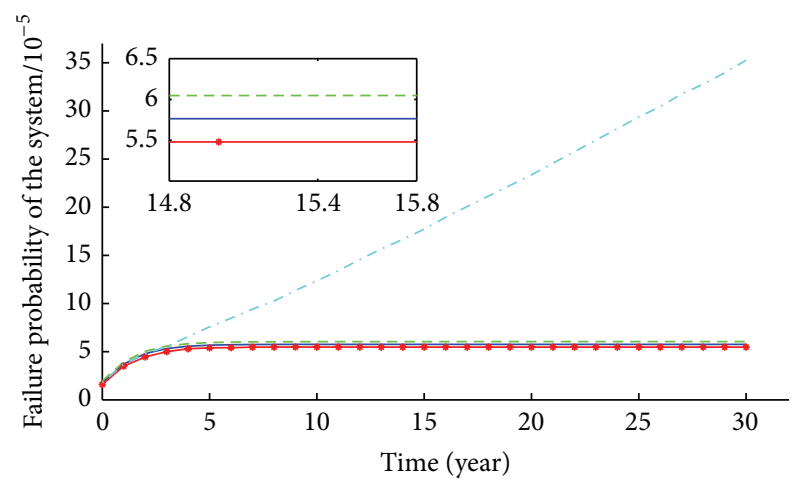

- The GO-FLOW method considering repair rate but not total signaling

- - - The GO-FLOW method considering repair rate and total signaling

* The dynamic fault tree method

-. - The GO-FLOW method considering neither repair rate nor total signaling

FIGURE 8: The failure probability's changing trend of NPP's offsite power system.

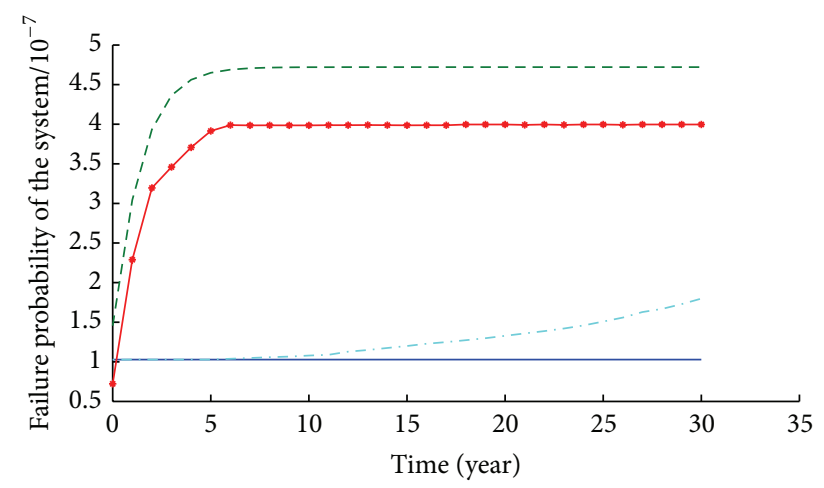

The GO-FLOW method considering repair rate but
not total signaling
-- The GO-FLOW method considering repair rate and
total signaling
- The dynamic fault tree method
The GO-FLOW method considering neither repair rate
nor total signaling

FIGURE 9: The failure probability's changing trend of NPP's power supply system.

(2) NPP's power supply system has complex structure and various states; meanwhile the total signaling has great influence on it. Thus, the failure probability of NPP's power supply system calculated by GOFLOW method considering repair rate but not total signaling is about $70 \%$ different from that calculated by GO-FLOW method considering repair rate and total signaling.

(3) When the repair rate and the total signaling are not taken into consideration, the failure probability of NPP's power supply system increases year by year with the operation time. When the repair rate and the total signaling are taken into consideration, within the five years after NPP is put into operation, the failure probability of NPP's power supply system increases obviously due to the increase of components' failure rate, while, five years later, the failure probability of NPP's power supply system gradually flattens out due to components' high repair rate.

(4) The failure probability of NPP's power supply system calculated by improved GO-FLOW method has the same time-varying trend with that calculated by DFT method. It demonstrates that the improved GOFLOW method can effectively analyze failure probability of NPP's power supply system.

(5) The failure probability of NPP's power system calculated by improved GO-FLOW method is about $15 \%$ higher than that calculated by DFT method. Since the fault tree method is approximately calculated by the minimal cut set, the influence caused by multiple-combination minimal cut sets' simultaneous appearing during the inclusion-exclusion calculation is ignored. The fault tree method mainly calculates second-order cut sets, ignoring the third-order or above cut sets which are hard to calculate because the occurring probability is small and they are strongly interconnected. Error accumulates when the system's states increase. In comparison, the failure probability calculated by improved GO-FLOW method can avoid the errors.

\section{Conclusion}

The improved GO-FLOW method has been applied to the reliability evaluation of NPP's power supply system in this article. Calculation analysis verified the effectiveness of improved GO-FLOW method. And the following conclusions can be achieved:

(1) On account of the NPP's power supply system, the GO-FLOW operators have been improved and improved GO-FLOW method for complex repairable system has been put forward. The improved GOFLOW model of NPP's power supply system has been established, with total signaling considered. Failure probability of NPP's offsite power system as well as power supply system has been simulated. The comparison analysis of the DFT method and the improved GO-FLOW method verified the accuracy and applicability of improved GO-FLOW method.

(2) In the reliability analysis of multimode complex repairable system based on the GO-FLOW method, the time-varying trend is linear neglecting the repair rate, which is not consistent with the actual case. When repair rate is introduced and total signaling is considered, the calculation results are much more accurate and reasonable.

(3) The operation model of NPP's power supply system has been achieved based on improved GO-FLOW method. Different from the DFT method, the model's scale does not expand with the increase of the system 
states in terms of GO-FLOW method. And the quantitative calculation results are about 10\% 15\% higher in comparison with the DFT method since there are no simplified approximations.

\section{Competing Interests}

The authors have declared that no conflict of interests exists.

\section{Acknowledgments}

This work was financially supported by the National Natural Science Foundation of China (51307123 and 51677137). The authors are grateful to experts in China Southern Power Grid for providing engineering data.

\section{References}

[1] P. Zhang and K. W. Chan, "Reliability evaluation of phasor measurement unit using Monte Carlo dynamic fault tree method," IEEE Transactions on Smart Grid, vol. 3, no. 3, pp. 1235-1243, 2012.

[2] Z. Sun and L. Li, "Potential capability estimation for real time electricity demand response of sustainable manufacturing systems using Markov Decision Process," Journal of Cleaner Production, vol. 65, no. 4, pp. 184-193, 2014.

[3] V. Pagonis, E. Gochnour, M. Hennessey, and C. Knower, "Monte Carlo simulations of luminescence processes under quasiequilibrium (QE) conditions," Radiation Measurements, vol. 67, no. 4, pp. 67-76, 2014.

[4] F. Zhao, L. Liang, and S. Wang, "A reliability evaluation of high speed railway overhead contact systems based on the GOFLOW methodology," Transactions of China Electrotechnical Society, vol. 30, no. 12, pp. 351-356, 2015.

[5] B. Gjorgiev, A. Volkanovski, D. Kančev, and M. Čepin, "Alternative off-site power supply improves nuclear power plant safety," Annals of Nuclear Energy, vol. 71, pp. 304-312, 2014.

[6] US Nuclear Regulatory Commission, NUREG/CR-6890 Vol 2-Reevaluation of Station Blackout Risk at Nuclear Power Plants: Analysis of Station Blackout Risk, US Nuclear Regulatory Commission, Washington, DC, USA, 2005.

[7] K. D. Rao, V. Gopika, V. V. S. Sanyasi Rao, H. S. Kushwaha, A. K. Verma, and A. Srividya, "Dynamic fault tree analysis using Monte Carlo simulation in probabilistic safety assessment," Reliability Engineering \& System Safety, vol. 94, no. 4, pp. 872883, 2009.

[8] H. Muhammad, Y. Hidekazu, M. Takeshi, and Y. Ming, "Common cause failure analysis of PWR containment spray system by GO-FLOW methodology," Nuclear Engineering and Design, vol. 262, pp. 350-357, 2013.

[9] M. Hashim, H. Yoshikawa, T. Matsuoka, and M. Yang, "Considerations of uncertainties in evaluating dynamic reliability by GO-FLOW methodology-example study of reliability monitor for PWR safety system in the risk-monitor system," Journal of Nuclear Science and Technology, vol. 50, no. 7, pp. 695-708, 2013.

[10] M. Hashim, Y. Hidekazu, M. Takeshi, and Y. Ming, "Application case study of AP1000 automatic depressurization system (ADS) for reliability evaluation by GO-FLOW methodology," Nuclear Engineering and Design, vol. 278, pp. 209-221, 2014.

[11] S. H. Lee, H. E. Kim, K. S. Son, S. M. Shin, S. J. Lee, and H. G. Kang, "Reliability modeling of safety-critical network communication in a digitalized nuclear power plant," Reliability Engineering and System Safety, vol. 144, pp. 285-295, 2015.

[12] Y. Liu and Y. Yu, "A T-H model for residual heat removal system reliability analysis," Energy Procedia, vol. 39, pp. 283-293, 2013.

[13] Y. Zou, L. Zhang, and P. Li, "Reliability forecasting for operators' situation assessment in digital nuclear power plant main control room based on dynamic network model," Safety Science, vol. 80, pp. 163-169, 2015.

[14] M. Hashim, H. Yoshikawa, T. Matsuoka, and M. Yang, "Quantitative dynamic reliability evaluation of AP1000 passive safety systems by using FMEA and GO-FLOW methodology," Journal of Nuclear Science and Technology, vol. 51, no. 4, pp. 526-542, 2014.

[15] M. Hashim, H. Yoshikawa, T. Matsuoka, and M. Yang, "Reliability monitor for PWR safety system using FMEA and go-flow methodology: application of risk monitor for nuclear power plants," in Proceedings of the 2013 21st International Conference on Nuclear Engineering (ICONE '13), Chengdu, China, August 2013.

[16] E. Zio, "Integrated deterministic and probabilistic safety assessment: concepts, challenges, research directions," Nuclear Engineering and Design, vol. 280, pp. 413-419, 2014.

[17] Z. Bai, X. Li, R. Tan, and B. Lian, "A function failure analysis method for improving reliability of the product based on GOFLOW methodology," in Proceedings of the IEEE International Conference on Industrial Engineering and Engineering Management (IEEM '08), pp. 550-555, Singapore, December 2008.

[18] A. C. G. Melo and M. V. F. Pereira, "Sensitivity analysis of reliability indices with respect to equipment failure and repair rates," IEEE Transactions on Power Systems, vol. 10, no. 2, pp. 1014-1021, 1995.

[19] T. Okazaki, N. Mitomo, and T. Matsuoka, "The use of the GO-FLOW methodology to investigate the aging effects in nuclear power plants (PSAM-0113)," in Proceedings of the 8th International Conference on Probabilistic Safety Assessment \& Management (PSAM '06), ASME Press, 2006.

[20] Z. Shen and X. Huang, The Principle and Application of the GO Method, Tsinghua University Press, Beijing, China, 2004.

[21] Northwest Power Design Institute, Electric Wiring And Distribution of Power Plants And Substations, Water Conservancy and Electric Power Press, Shanxi, China, 1982. 

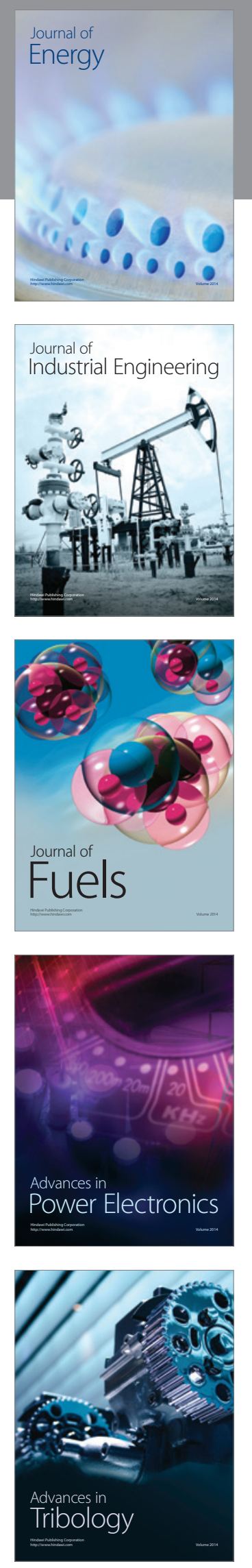
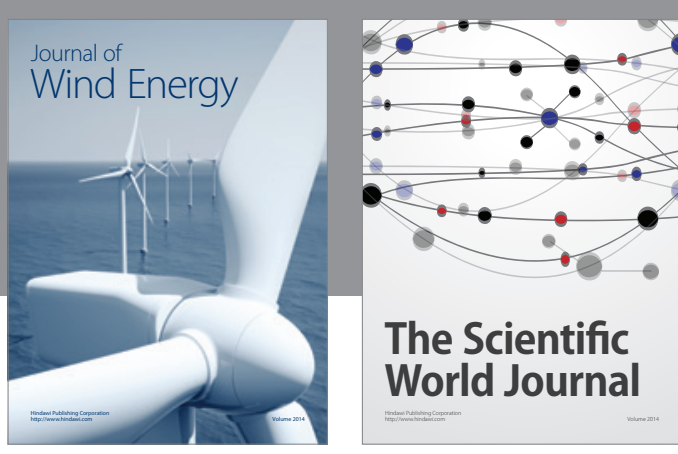

The Scientific World Journal
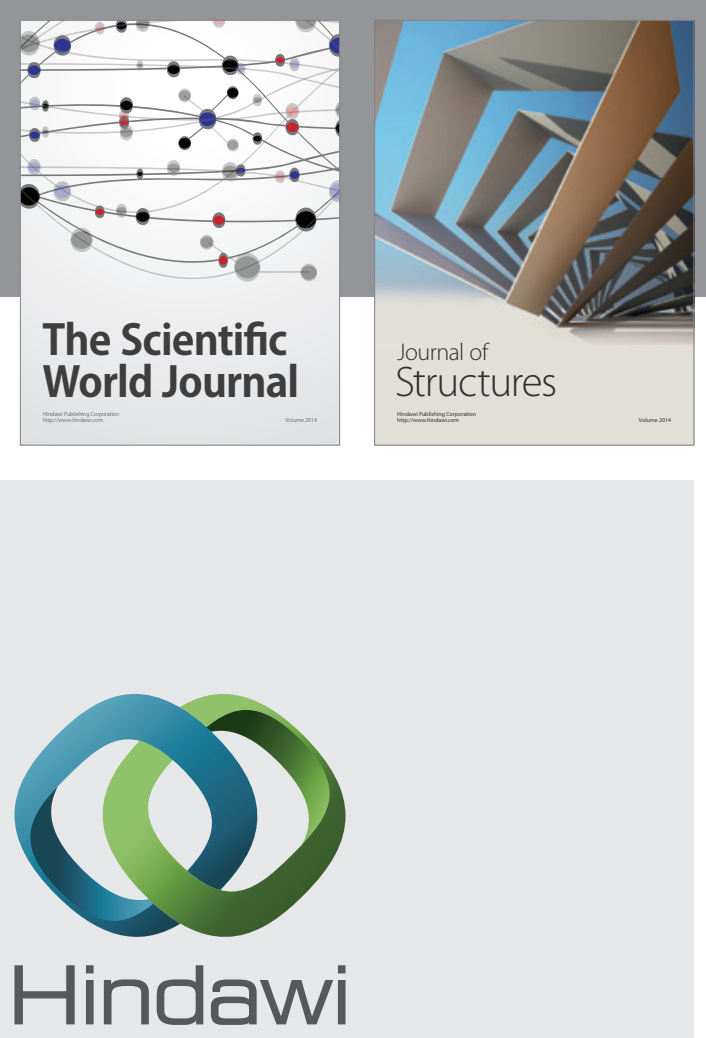

Submit your manuscripts at

http://www.hindawi.com
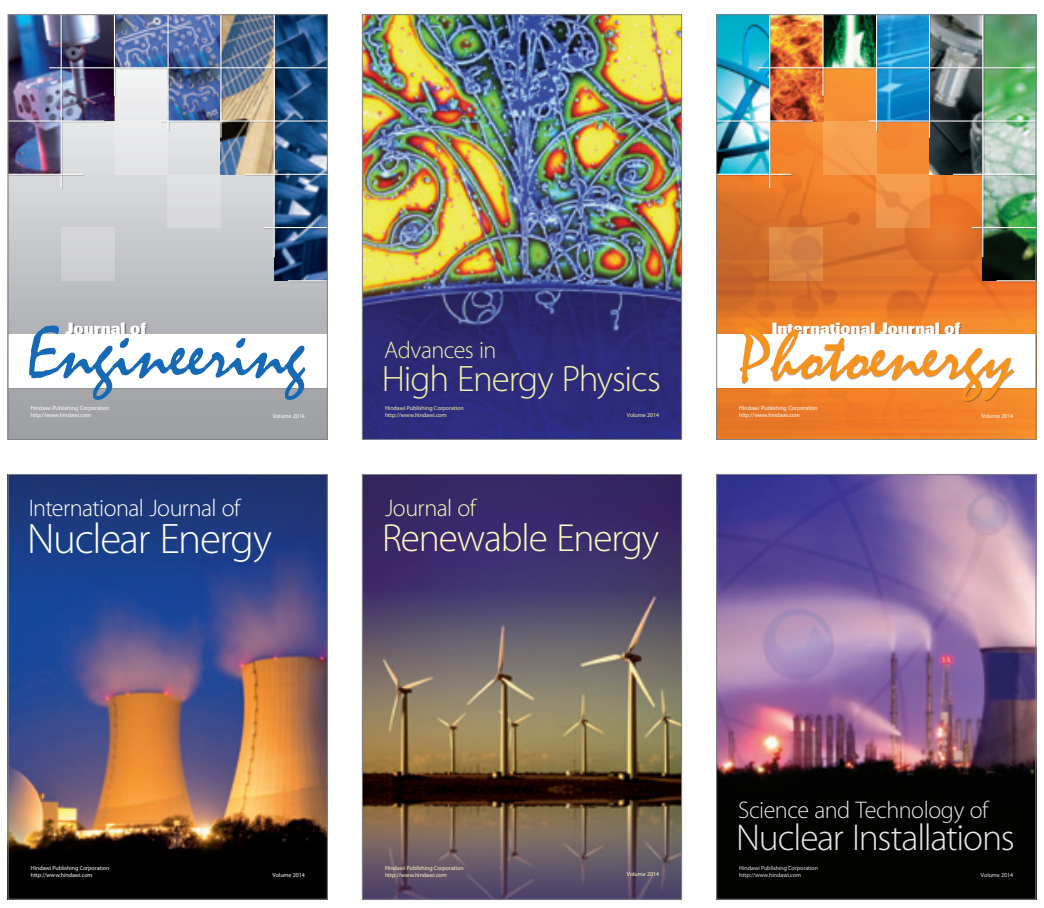
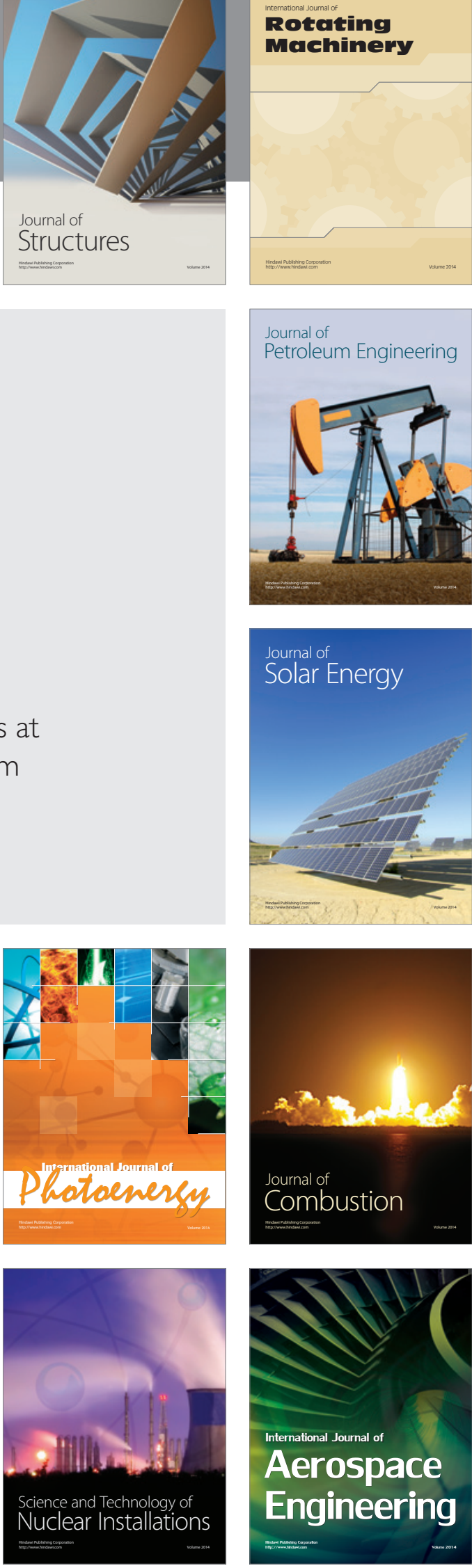